\title{
Genetics of GNE myopathy in the non-Jewish Persian population
}

\author{
Alireza Haghighi ${ }^{\star 1,2,3}$, Shahriar Nafissi ${ }^{\star, 4}$, Abrar Qurashi $^{5}$, Zheng Tan ${ }^{5}$, Hosein Shamshiri ${ }^{4}$, Yalda Nilipour ${ }^{6}$, \\ Amirreza Haghighi ${ }^{7}$, Robert J Desnick ${ }^{5}$ and Ruth Kornreich ${ }^{\star, 5}$
}

GNE myopathy is an autosomal recessive adult-onset disorder characterized by progressive muscle atrophy and weakness, initially involving the distal muscles, while often sparing the quadriceps. It is caused by variants in the GNE gene that encodes a key bifunctional enzyme in the sialic acid biosynthetic pathway. We investigated the clinical and molecular characteristics of 18 non-Jewish Persian patients from 11 unrelated GNE myopathy families. In addition, we reviewed the previously reported cases and suggest genotype-phenotype correlations for the identified variants. Comprehensive clinical and laboratory evaluations were carried out. Sequencing of the GNE gene was performed using genomic DNA from the patients. Screening of the identified variants was performed in all relevant family members. Molecular analyses identified three causative homozygous GNE variants in 11 families: c.2228T $>$ C (p. M743T) in 7, c.830G $>$ A (p.R277Q) in 2, and one novel variation (c.804G $>$ A) in 2 families that results in a synonymous codon change $(p \cdot L 268=)$ and likely creates a novel splice site affecting the protein function. This study confirms that c.2228T $>C$ (p.M743T) is the most prevalent disease-causing variant in the non-Jewish Persian population, but other GNE variants can cause GNE myopathy in this population. The patients with all three different variants had similar ages of onset. The youngest patient was an 18-year-old girl in whom the c.830G $>$ A (p.R277Q) variant was identified, whereas the oldest onset age (31 years) was seen in a male patient with c.804G $>$ A (p.L268 =). The results of this investigation expand our knowledge about the genotype-phenotype correlations in GNE myopathy and aid in clinical management and therapeutic interventions.

European Journal of Human Genetics (2016) 24, 243-251; doi:10.1038/ejhg.2015.78; published online 13 May 2015

\section{INTRODUCTION}

GNE myopathy, also known as hereditary inclusion body myopathy (HIBM or IBM2) and distal myopathy with rimmed vacuoles (DMRV), is a rare young adult-onset non-inflammatory progressive disabling myopathy that is inherited as an autosomal recessive trait. The disease, first described in four Jewish families of Persian descent, ${ }^{1}$ is characterized by onset of distal muscle weakness of the lower limbs, especially the tibialis anterior muscles, which progresses to the proximal muscles. Notably, the quadriceps, for unknown reasons, is selectively spared, despite marked weakness of all other hip muscles. ${ }^{2}$ Upper limbs, especially the scapular and proximal muscles, are usually involved in the advanced stages. ${ }^{1}$ Typically, the initial disease manifestation is foot drop and the consequent altered gait resulting from the involvement of the foot dorsiflexion muscles. As the disease progresses patients become wheelchair bound $\sim 10-12$ years after onset. $^{3}$

The typical histopathological findings in GNE myopathy include cytoplasmic rimmed vacuoles and congophilic inclusions mainly in angular atrophic fibers, some arranged in groups using Gomori trichrome and Congo red stains. Presence of necrotic or basophilic degenerative fibers and endomysial fibrosis are not among usual findings but can be seen in typical cases. Chronic inflammatory cell infiltration is less frequent and is a distinguishing histopathologic feature from sporadic inclusion body myositis. ${ }^{4-6}$ The presence of diagnostic findings in biopsy depends on the site of muscle biopsy. Sometimes choosing the site of biopsy is a challenge because distal muscles could be completely wasted and proximal muscles are spared.

GNE myopathy results from variants in the GNE gene, ${ }^{7,8}$ which encodes the bifunctional rate-limiting enzyme, UDP- $N$-acetylglucosamine (UDP-GlcNAc) 2-epimerase/ $N$-acetylmannosamine (ManNAc) kinase. The GNE encoded enzyme consists of 722 amino acids and possesses two functional domains: an N-terminal epimerase domain (encoded by exons 2-6) and a C-terminal kinase domain (encoded by exons 7-12). ${ }^{9,10}$ This enzyme plays a key role in the biosynthesis of $\mathrm{N}$-acetylneuraminic acid, a member of the sialic acid family, catalyzing the rate-limiting initial two steps of the cytosolic synthesis of $\mathrm{N}$-acetylneuraminic acid from UDP-GlcNAc. ${ }^{9,10}$ Sialic acids are the most ubiquitous terminal monosaccharides on complex glycoproteins

\footnotetext{
${ }^{1}$ Department of Genetics, Harvard Medical School, Boston, MA, USA; ${ }^{2}$ Department of Medicine and the Howard Hughes Medical Institute, Brigham and Women's Hospital, Boston, MA, USA; ${ }^{3}$ Wellcome Trust Centre for Human Genetics, University of Oxford, Oxford, UK; ${ }^{4}$ Department of Neurology, Iranian Center of Neurological Research, Tehran University of Medical Sciences, Shariati Hospital, Tehran, Iran; ${ }^{5}$ Department of Genetics and Genomic Sciences, Icahn School of Medicine at Mount Sinai, New York, NY, USA; ${ }^{6}$ Department of Pathology, Pediatric Pathology Research Center, Mofid Children Hospital, Shahid Beheshti Medical University, Tehran, Iran; ${ }^{7}$ Toronto General Hospital, University of Toronto, Toronto, Canada

${ }^{*}$ Correspondence: Dr A Haghighi, Department of Genetics, Harvard Medical School, 77 Ave Louis Pasteur, Boston, MA 02115, USA. E-mail: haghighi@genetics.med.harvard.edu, or haghighimd@yahoo.com

or Dr S Nafissi, Department of Neurology, School of Medicine, Iranian Center of Neurological Research, Tehran University of Medical Sciences, Shariati Hospital, North Karegar Street, Tehran 14114, Iran. E-mail: nafisi@sina.tums.ac.ir

or Dr R Kornreich, Department of Genetics and Genomic Sciences, Box 1497, Icahn School of Medicine at Mount Sinai, One Gustave L. Levy Place, New York, NY 10029, USA. Tel: +1 212241 1979; Fax: +1 212241 1464; E-mail: ruth.kornreich@mssm.edu

Received 26 December 2013; revised 27 December 2014; accepted 7 January 2015; published online 13 May 2015
} 
and glycolipids, which are located on the surface of eukaryotic cells, with important roles in biological recognition processes, including cell-cell interactions and binding of cellular receptors to their target bacteria, viruses, parasites, and toxins. ${ }^{11}$ In addition, sialic acids play important roles in determining the survival time and biologic activity of various glycoproteins, hormones, and enzymes, in vivo. ${ }^{12}$

The GNE gene, located on chromosome 9p12-13 is $\sim 62.6 \mathrm{~kb}$ and consists of 13 exons encoding multiple GNE protein isoforms, its longest consisting of 753 amino acids. GNE is expressed in almost all body tissues but the highest expression has been observed in the liver. 9,12 Interestingly, the tissues with the highest GNE expression, including liver, lung, and kidney, remain intact in GNE myopathy and the disease selectively affects skeletal muscles, which have relatively low levels of the enzyme. ${ }^{13}$

In the mouse, GNE is expressed very early and during all stages of development. ${ }^{13}$ Studies showed that the targeted inactivation of GNE in early mice embryos is lethal. ${ }^{14}$ These observations suggest an important role for this enzyme in development.

GNE also plays an important role in cell regulation through interaction with two important regulatory proteins, collapsin response mediator protein 1 and the promyelocytic leukemia zinc-finger protein. ${ }^{15}$

The exact pathogenesis of GNE myopathy remains unknown. It has been shown that normal sialylation is crucial for the stabilization and function of skeletal muscle glycoproteins and that modifications in the sialylation of cell surface glycoproteins can influence cell adhesion and signal transduction and cause myofibrillar degeneration, resulting in loss of normal muscle function. ${ }^{16-18}$ Studies have demonstrated that some skeletal muscle proteins, such as neprilysin, ${ }^{19}$ alphadystroglycan, ${ }^{16} \mathrm{O}$-linked glycans, ${ }^{20}$ and neural cell adhesion molecule $(\mathrm{NCAM})^{17}$ are hyposialylated in GNE myopathy, a distinctive feature in comparison with other myopathies with similar clinical manifestations. The suggested impact of GNE variants on sialylation remains controversial and under study.

Here, we investigated the clinical features and molecular basis of GNE myopathy in 18 patients from 11 affected families from the nonJewish Persian population.

\section{PATIENTS AND METHODS}

\section{Patients}

Eighteen HBIM patients from 11 families from the Iranian non-Jewish population were identified. The families were mainly from central and southern regions of Iran (Figure 1). The diagnosis of GNE myopathy was based on the presence of slowly progressive myopathy with a characteristic distribution of weakness (with prominent involvement of distal muscles and relative quadriceps sparing) and positive biopsy findings (presence of rimmed vacuoles).

Written consents for participation in clinical and molecular studies were obtained from all family members. The study was conducted in accordance with the Declaration of Helsinki.

\section{Methods}

Genomic DNA was extracted from peripheral blood of all patients, their parents and healthy siblings, using QIAamp DNA blood Midi kits (Qiagen, Hilden, Germany).

Muscle biopsies were taken from 11 patients, at least one affected member from each family, for histology and immunohistochemistry studies.

Affected members of each family were first tested for the Persian Jewish GNE founder variant, c.2228T >C (p.M743T) by targeted allele-specific primer extension analysis. For exon sequencing of the GNE gene, genomic DNA was PCR amplified and the resultant amplicons were then sequenced using ABI PRISM Big Dye Terminator Cycle Sequencing (Applied Biosystems, Grand Island, NY, USA) on an ABI 3730xl automated sequencer (Applied Biosystems;

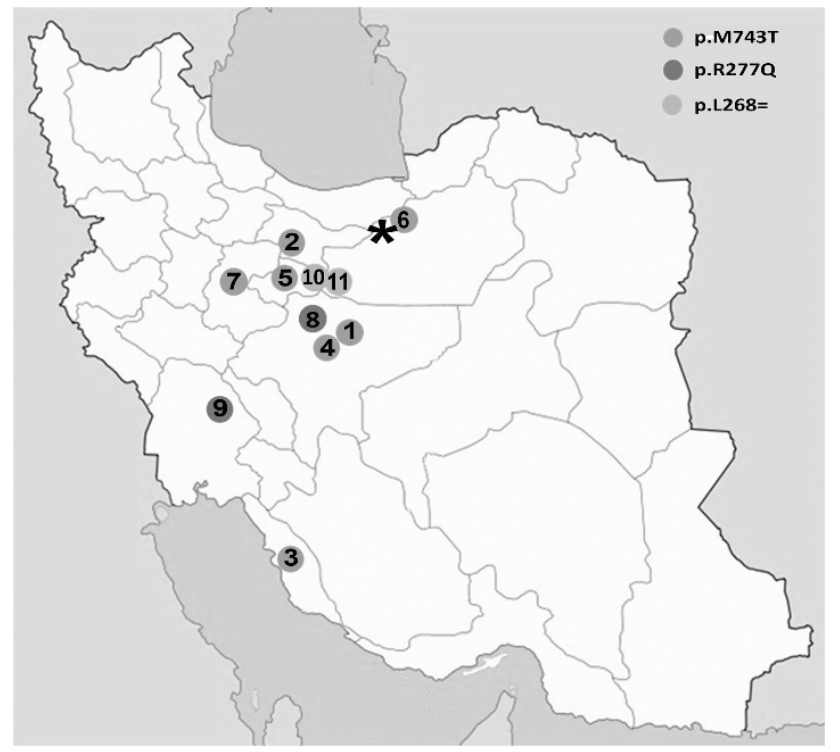

Figure 1 Geographical distribution of GNE variants in Iran. Numbers in the map represent the family numbers in Table 1 . The black asterix shows the location of Sangsar.

for details of methods see Supplementary Methods). NP_001121699 was used to denote amino acid changes in hGNE2 and NM_001128227 for nucleotide changes in mRNA variant 1 .

The variants and associated phenotypes were submitted to ClinVar (www.clinvar.com).

\section{RESULTS}

\section{Clinical findings}

Eighteen patients from 11 Muslim Persian families: 9 (50\%) female and $9(50 \%)$ male, were examined at hospitals of Tehran University of Medical Sciences. Details of the clinical findings are shown in Table 1. The mean age at examination and mean onset age of our patients were 34 (24-47) and 25.7 (19-31) years, respectively. Nine (50\%) patients from seven families had consanguineous parents. The parents of the patients were healthy. Most (67\%) of our patients presented with symptoms indicative of distal lower extremity weakness such as steppage gait or tiptoe walking, whereas the main chief symptom in the others was a gait disturbance due to hip-girdle weakness. One family (10a, b, and c) had hand weakness from the beginning. Pain or muscle cramping was not a major symptom in any of the patients and sensory symptoms were generally absent. In the majority of our patients (94.5\%), the quadricep muscles were relatively spared. Magnetic resonance imaging (MRI) of thigh and leg muscles of patient $7 \mathrm{~b}$ is shown in Figure 2. In the lower extremities, hip adductors, flexors, foot dorsiflexors, and knee flexors were frequently involved, and hip abductors and plantar flexors were involved in lesser degrees. Most patients (94.5\%) had some level of upper limb involvement, except for one patient ( $7 \mathrm{a}$, aged 47 years). Weakness usually started from intrinsic hand muscles with involvement of deep finger flexors in some patients. Elbow flexors and extensors became weak later in the disease course. Three $(17 \%)$ of our patients were wheelchair bound and four others (22\%) used wheelchairs most of the time. Facial, ocular, and bulbar muscles were generally spared and none of our patients had scapular winging or spinal deformities. Neck flexor muscles were only involved in three $(17 \%)$ wheelchair bound patients (10a, 10c, and 9a, all over 40 years of age), a finding that 


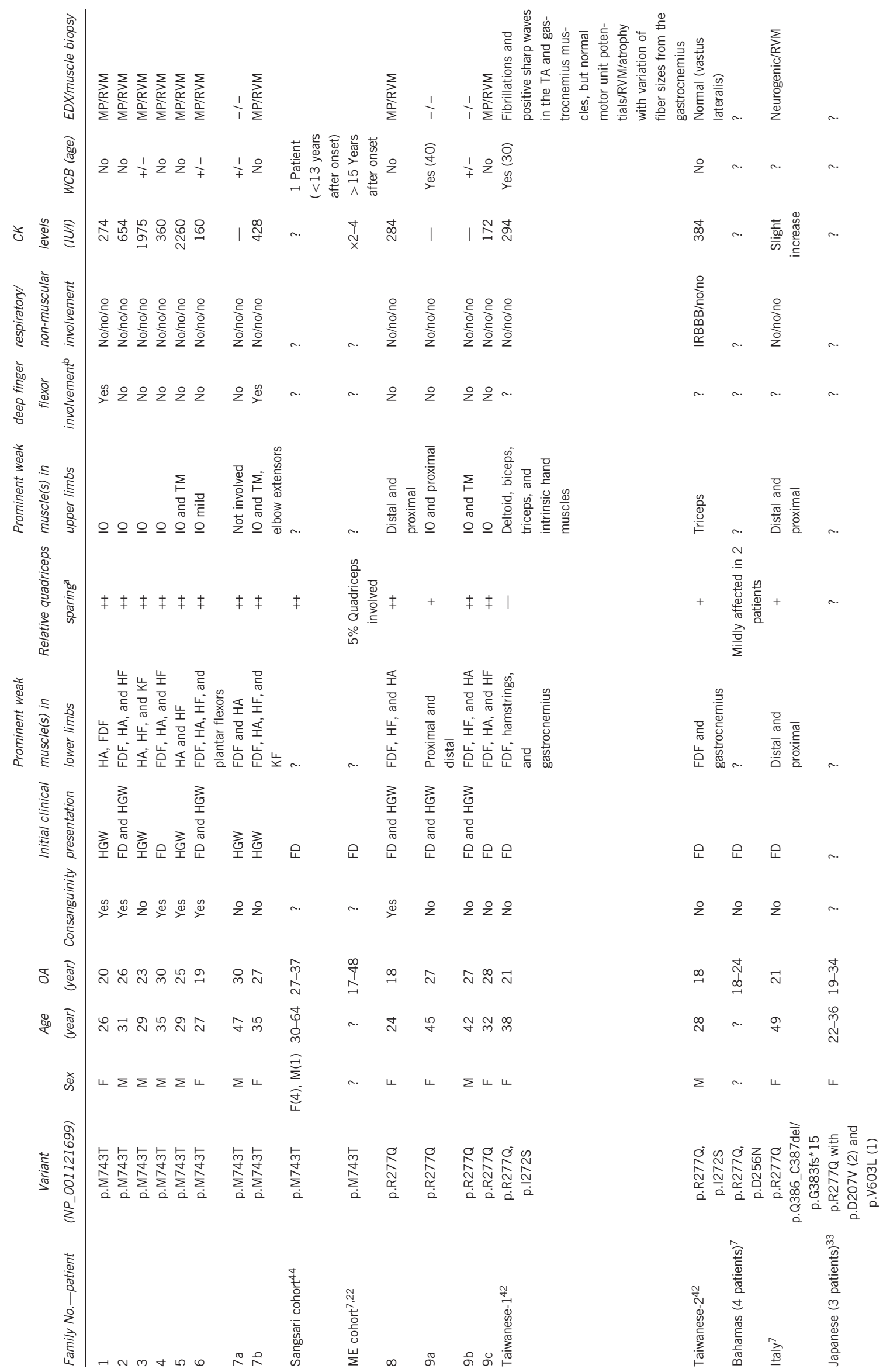


probably emerges in later stages. Our patients did not have any cardiac, respiratory, or other non-muscular involvement.

Electromyography (EMG) revealed myopathic changes in the examined muscles of our patients. Active denervation in the form of fibrillation potentials and positive sharp waves were all prominent electrophysiologic findings in more involved muscles and nine patients who had EMG, had active denervation in their tibialis anterior muscles. Serum creatine kinase (CK) levels were either normal or mildly elevated in some patients, but usually below $1000 \mathrm{IU} / \mathrm{l}$. Only five patients had serum CK levels from 1338-5300 IU/l, especially in the early stages of the disease. Electrocardiography (ECG), echocardiography, and pulmonary function tests were performed in all patients and did not reveal any abnormality.

\section{Pathological findings}

Histological and histochemical studies of muscle biopsies taken from selected muscles in patients (one sample in each patient) support the diagnosis of rimmed vacuolar myopathy. Histological findings were remarkably variable and were composed of mixed myopathic and neurogenic features. The number of rimmed vacuoles ranged from very few to many and the appearance of the vacuoles varied from small cleft like to large round or irregular structure. Typical necrosis/ regeneration was not seen, but endomysial fibrosis was noted in prominently affected fascicles (Figures $3 \mathrm{a}$ and b). Interestingly, neurogenic features were variable from small clusters of angulated fibers resembling an adult case of motor neuron disease to a prominent fascicular atrophy resembling Werdnig-Hoffmann disease on haematoxylin and eosin stain (Figures $3 c$ and $d$ ). The details of pathologic findings are elaborated in Table 2.

\section{Molecular analysis}

Molecular analyses of GNE in the 11 families diagnosed clinically with GNE myopathy identified all families carrying variants that affect or are likely to affect the protein fuction. Targeted variant analysis for the known GNE myopathy founder variant, c.2228T >C (p.M743T), revealed that eight patients from seven families were homozygous for the lesion. This variant alters the methionine at position 743 to threonine in exon 13 (NCBI Reference Sequence: NG_008246.1) and is within a kinase domain. We also identified four patients from two families carrying the homozygous missense variant, c.830G $>$ A. This variant in exon 5 replaces an arginine with a glutamine at position 277 in the epimerase domain, c.830G $>$ A (p.R277Q). In addition, a synonymous variant c.804G $>$ A $($ p.L268 $=)$ in exon 5 (NCBI Reference Sequence: NG_008246.1) was identified in six patients from two families. Although this variant was deep into the exon and did not alter a codon, there is evidence that the variant may be causative as it segregated with the disease as the parents were heterozygous and healthy siblings were either heterozygous or wild type for the identified variant whereas the affecteds were homozygous for the change. The sequence surrounding the variant is shown in Supplementary Figure 1a. This synonymous exonic variant most likely causes a splice site aberration, as revealed by the Human Splicing Finder (HSF) program (http://www.umd.be/HSF/) (Supplementary Figure 1b). The HSF calculated consensus values (CV) of potential splice sites and branch points, and predicted this variant to be 'probably damaging' creating a potential splice site. ${ }^{21}$ This finding was confirmed by MutationTaster (http://www.mutationtaster.org/), another software application, which predicted this variant to be probably damaging and placed the variant in the 'affect protein function' class. 

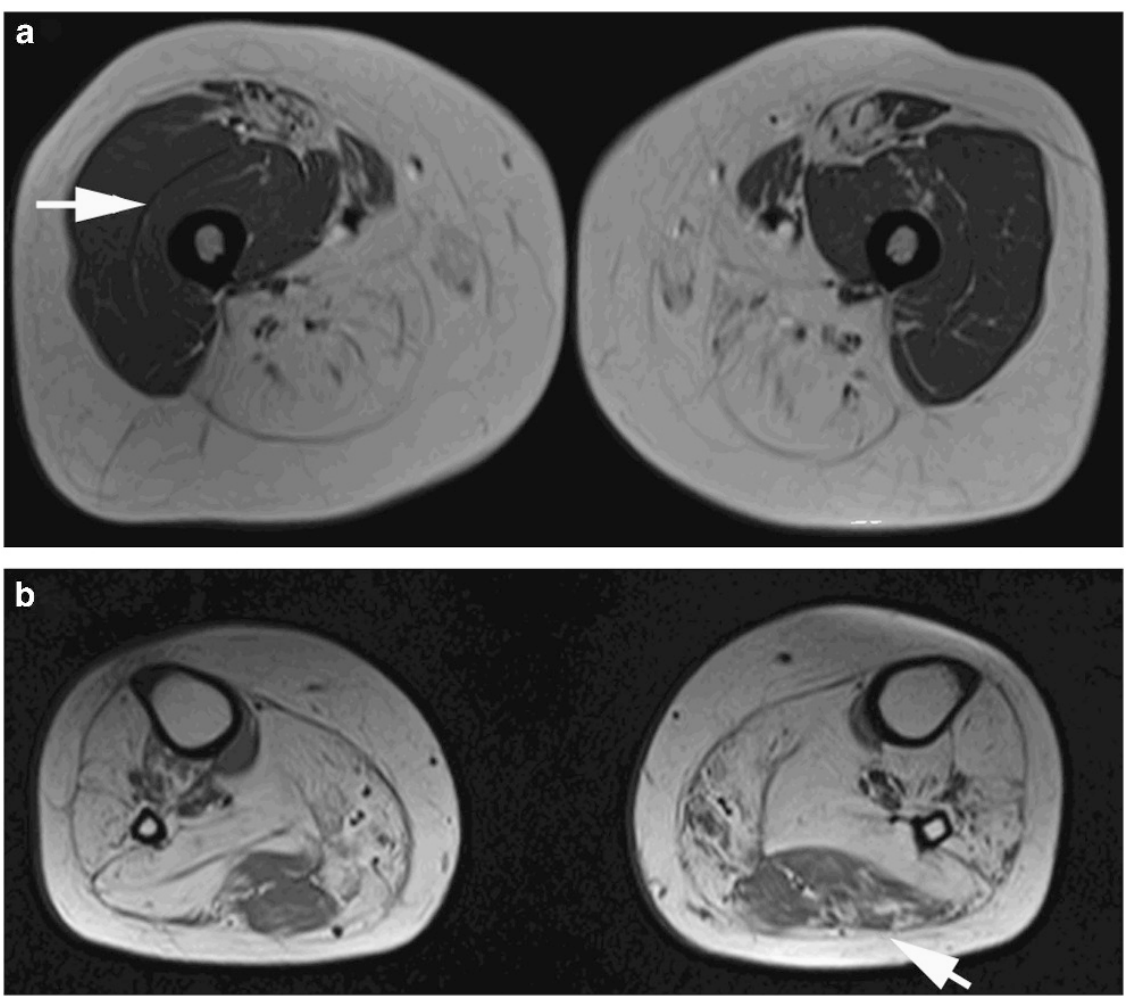

Figure 2 Thighs (a) and legs (b) T1-weighted MR images (patient 7b). (a) Atrophy and fat replacement of posterior thigh muscles with relative sparing of quadriceps (white arrow) and sartorius muscles. (b) Fatty replacement in anterior and posterior leg muscles with relatively less involvement of the lateral head of gastrocnemius muscle (white arrow).

\section{Phenotype spectrum of variants}

To date, no genotype-phenotype correlation has been described in GNE myopathy. Patients with the same GNE genotype may have very different clinical manifestations, not fully explained. The results of this study have expanded the phenotypic spectrum associated with variants in the GNE gene.

In our study, the mean 25 years of age at onset in patients with the homozygous c.830G > A (p.R277Q) variant was similar to those for patients homozygous for the c.2228T $>$ C (p.M743T; 26.75 years) or c. $804 \mathrm{G}>$ A (p.L268 $=; 27.1$ years) variants. The average age of onset of Middle-Eastern patients homozygous for the c.2228T $>$ C (p.M743T) variant (based on data from 140 patients) was 30 years. ${ }^{7,22}$ The earliest and the latest recorded age of onset in patients from the Middle East were 17 and 48 years, respectively. 5,17,23,24 The mean onset age of Japanese and Indian patients was 26 years. ${ }^{5,20}$ The average onset age in our 18 patients was similar, 25.7 years.

Three of our c.2228T $>$ C (p.M743T) patients who were $>6$ years after the onset used wheelchairs, but none of them was wheelchair bound, even 17 years after the GNE myopathy onset (7a, 47 years; Table 1). The Middle Eastern patients harboring c.2228T $>C$ (p.M743T) became wheelchair bound on average 15 years after the disease onset, ${ }^{5}$ whereas ambulatory loss occurred earlier in Indian (3-9 years after the disease onset $)^{25}$ and Japanese $(12 \text { years })^{3}$ patients. Of our patients homozygous for c.830G > A (p.R277Q; Table 1), one (9b, 42 years) used a wheelchair intermittently (15 years after onset) and another $(9 \mathrm{a}, 45)$ became wheelchair bound 13 years after onset.

Very recently, Khademian et al. investigated the prevalence of c.2228T > C (p.M743T) variant in a relatively isolated community of Northern Iran, from Sangsar (also known as Mahdishahr; $35^{\circ} 42^{\prime} 39^{\prime \prime}$
$\mathrm{N}$ and $\left.53^{\circ} 21^{\prime} 14^{\prime \prime} \mathrm{E}\right)$. The frequencies of heterozygous and homozygous c.2228T $>$ C (p.M743T) variant in Sangsar were 3.91 and $0.63 \%$, respectively; ${ }^{26}$ which are similar to those of Persian Jewish population. ${ }^{27}$ Sangsar is only $6 \mathrm{~km}$ away from Shahmirzad, a city with many Jewish families. It is estimated that a subset of the present day Sangsari population is of relatively recent Jewish descent, because marriages of individuals from these two cities is very common, however, no haplotype analysis has been performed to confirm this. The average age of onset in the Sangsari patients was 32 years, similar to that of the Middle-Easterners homozygous for the c.2228T $>\mathrm{C}$ (p.M743T) variant. Three out of five Sangsari patients (female, 32, 40, and 64 years) had only difficulty in walking and only one patient (40 years) was wheelchair bound. The fifth individual (male) homozygous for c.2228T >C (p.M743T) did not have any obvious myopathy symptoms. None of our families were of Jewish ethnicity or from cities with a significant Jewish population, and only one of our patients (6, Table 1) was from an area close to Sangsar.

In most GNE myopathy cases, serum CK was elevated. ${ }^{28}$ The serum CK level in our patients ranged from 160 to $2260 \mathrm{IU} / \mathrm{l}$ in cases homozygous for c.2228T >C (p.M743T), a kinase domain variant, whereas 172-274 IU/l in patients with the epimerase domain variant, c.830G > A (p.R277Q). Patients harboring the c.804G >A (p.L268 =) variant had CK levels ranging from 200 to $608 \mathrm{IU} / \mathrm{l}$. Very high CK levels $(>1300 \mathrm{IU} / \mathrm{l})$ were observed in younger patients who had a mean age of onset of 18.5 years.

Several atypical features have been identified in GNE myopathy patients, including sparse reports of major quadriceps involvement, ${ }^{5}$ lacking distal weakness, ${ }^{5}$ or limb-girdle myopathy. ${ }^{29}$ Incomplete penetrance of the disease has also been described; three individuals 

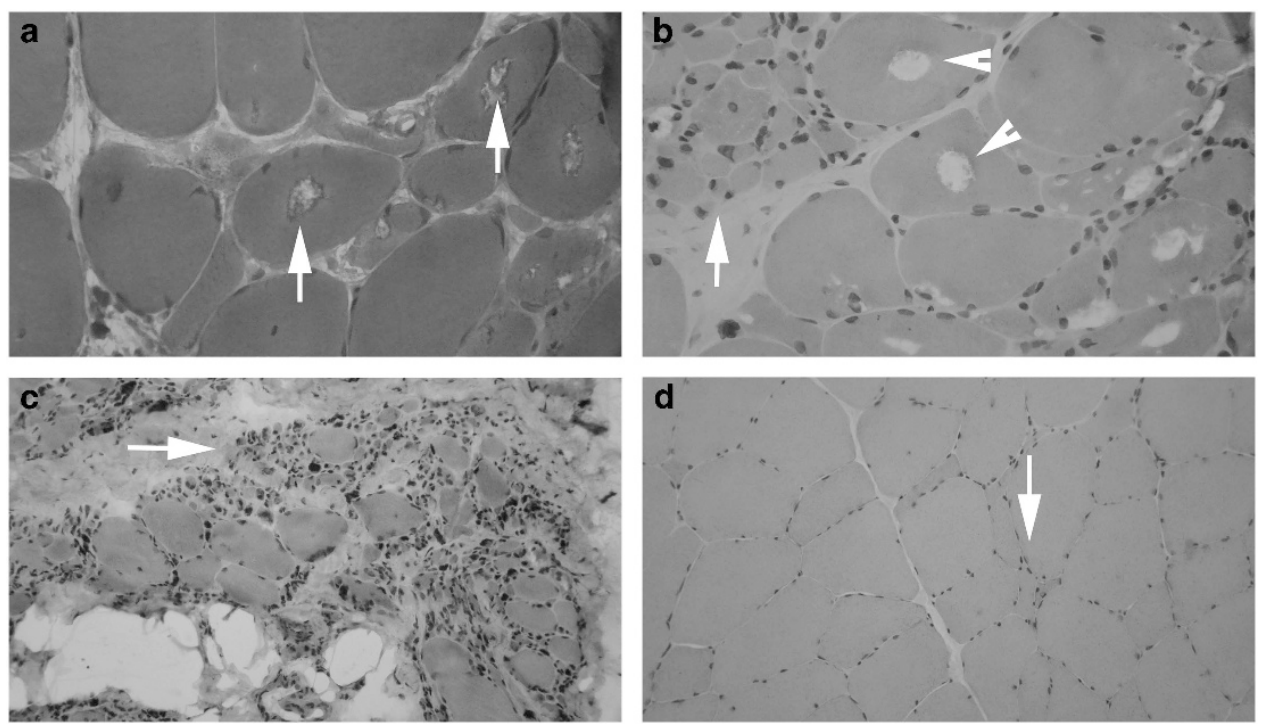

Figure 3 Pathological characteristics of GNE myopathy. (a) Red-rimmed cytoplasmic vacuoles showed by arrows (Gomori trichrome, high power, patient 9c muscle biopsy from right anterior tibialis). (b) Group atrophy (arrow) associated with prominent cytoplasmic vacuoles (arrowheads; H\&E, high power, patient 9c muscle biopsy from right anterior tibialis). (c) Large group atrophy (arrow) resembling 'spinal muscular atrophy' (H\&E, medium power, patient 11b muscle biopsy from left biceps). (d) Dispersed or small group of angulated atrophic fibers (arrow) resembling 'neurogenic atrophy' (H\&E, high power, patient 8 muscle biopsy from left biceps). The full colour version of this figure is available at European Journal of Human Genetics online.

(two Muslim Arabs and one Persian homozygous for c.2228T $>\mathrm{C}$ (p.M743T), and one Japanese homozygous for p.D176V variant), who still remained asymptomatic in their sixth to seventh decades of life. ${ }^{5,24,26}$ Interestingly, these three individuals were all female. Unusual biopsy findings of perivascular, perimysial, or endomysial inflammation have also been reported. ${ }^{4,5}$

Full involvement of quadriceps has been observed in 5\% of Middle Eastern patients, ${ }^{5,20}$ with onset age of 19-35 years. ${ }^{5}$ These patients became wheelchair bound earlier (5-8 years after onset). ${ }^{5}$ The involvement of quadriceps muscles was seen only in patients with at least one variant in the epimerase domain, ${ }^{12,30-32}$ whereas patients with a homozygous variant in the kinase domain did not have quadriceps involvement; suggestive of a variant-dependent pattern for muscle involvement. Quadriceps involvement was observed only in one patient $(5.5 \%)$ in this study, patient 9a who was homozygous for epimerase domain variant, c.830G >A (p.R277Q; however, not as severely involved as other pelvic girdle muscles), but not in patients with c.2228T >C (p.M743T), supporting this hypothesis. Studies in GNE myopathy patients have shown that the epimerase activity in lymphocytes is decreased, indicative of the partial functional loss of enzyme activity. ${ }^{24}$

Lack of distal weakness (even 5 years after disease onset) has been reported in two GNE myopathy patients from a Persian Jewish family homozygous for the c.2228T >C (p.M743T) variant. CT and MRI investigations revealed significant atrophy of the posterior hip compartment, and relative sparing of the quadriceps muscles. Distal muscles were normal on CT but had increased MRI signals on T2 in the tibialis anterior region. All patients in this study showed distal weakness.

Limb-girdle myopathy, instead of distal myopathy was very recently described in six Korean patients, ${ }^{29}$ three being wheelchair bound. The age of onset in these patients was 16-21 years (mean 19.3 \pm 1.8 ). Their disease started with weakness of the proximal part of the lower limbs and a waddling gait. CT scan showed involvement of hamstring and biceps brachii muscles, whereas the anterior tibial muscles were relatively spared. The GNE variants identified in these patients included p.V603L, p.C44S, p.G576Efs9*, p.M292V, and p.A662V. A recent study on Korean patients has reported that both homozygous p.V603L and p.C44S can cause distal myopathy as well. The mean onset age for these patients were 23.2 (p.V603L) and 30 (p.C44S) years. ${ }^{33}$

Cardiac involvement has been reported in a number of GNE myopathy patients, with a higher prevalence (18\%) in Japanese patients. ${ }^{28}$ Moreover, a recent animal study revealed the important role of GNE in the early development of cardiac muscles. ${ }^{34}$ Interestingly, none of our patients nor other reported Iranian non-Jewish patients $^{4,22}$ had any heart problems.

Facial weakness is another atypical feature in GNE myopathy that was reported ${ }^{5}$ in three patients in a Jewish and a Karaite family from the Middle East who were homozygous for c.2228T $>$ C ( $p$. $\mathrm{M} 743 \mathrm{~T}$ ). This abnormality was not present in the patients of this study.

Prominent involvement of deep finger flexors is typically seen in sporadic inclusion body myopathy. ${ }^{35}$ Interestingly, none of our patients with c.830G >A (p.R277Q; in the epimerase domain) exhibited out-of-proportion involvement of deep finger flexors, whereas two patients with c.2228T $>C$ (p.M743T) and two with the c.804G $>$ A (p.L268 = ) variant had prominent involvement.

\section{DISCUSSION}

GNE myopathy has been identified worldwide and in populations of diverse ethnicities, but the highest prevalence has been reported in the Persian Jewish population (1:1500) with a disease gene carrier frequency of $1-9 \% .{ }^{27,36}$ The prevalence of the disease in Japan (where the phenotype is often referred to as Nonaka myopathy) has been estimated at 1 in 1 million individuals. ${ }^{8}$ To date, around 150 GNE myopathy cases of Persian Jewish descent have been reported. ${ }^{7,22}$

Here, we studied 11 non-Jewish families from Iran affected with GNE myopathy and performed comprehensive clinical and molecular investigations of the 18 affected patients. Two disease-causing 


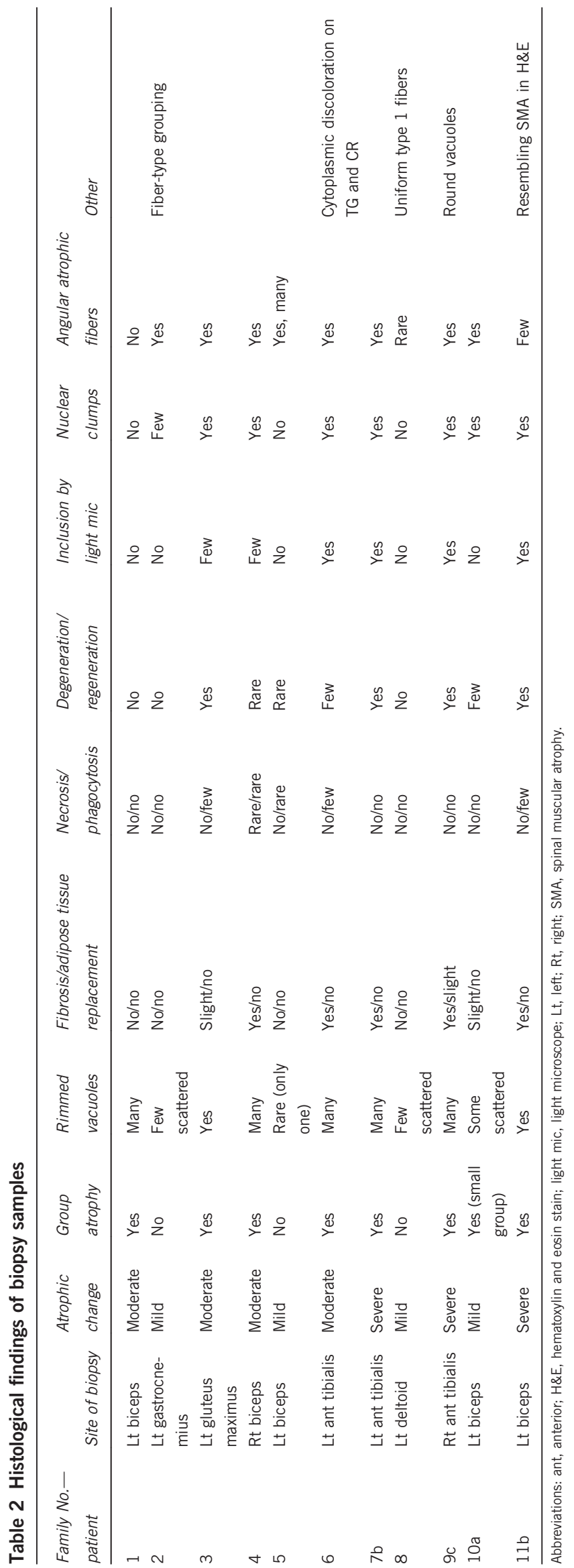

homozygous GNE missense variants, c.830G $>$ A (p.R277Q) and c.2228T >C (p. M743T) were identified to be responsible for the disease in nine families. We also identified one novel variant (c.804G > A) among six patients from two families, which leads to a synonymous codon change (p. L268L) and likely disrupts normal splicing. We were unable to obtain fresh blood or other samples for RT-PCR studies to clearly demonstrate the RNA defect.

It is likely that the c.2228T $>\mathrm{C}$ (p.M743T) variant was of nonJewish origin, particularly as it was present in the non-Jewish population. It likely entered the small Jewish population and became prevalent. A similar transfer of the common Jewish type 1 Gaucher disease variant, p.N409S, has been shown to have entered the Ashkenazi Jewish population from non-Jewish Europeans where it is also frequent. ${ }^{37}$ Since the c.2228T $>$ C (p.M743T) variant is found in the general non-Jewish population, it is likely that it and other rare variants occurred in the non-Jewish population and that the homozygous patients' parents are distant relatives, but they are unsure of prior multigenerational relationships, particularly because the parents are from the same town.

All three variants were homozygous in the patients of this study, whereas only seven families were consanguineous. Information about the allele frequency and distribution of these variants may explain the identification of homozygous patients in non-consanguineous families, however, such data are not available in Iran. In addition, the high rate of consanguinity in the general population in Iran might also contribute to homozygosity in patients 'not clearly known' to be consanguineous.

The homozygous variant (c.2228T > C, p.M743T) is the founder variant in Persian Jewish GNE myopathy cases. ${ }^{7}$ In patients from other ethnicities, several variants have been identified, most of which are compound heterozygotes for missense variants. ${ }^{30,31}$ The c.2228T >C (p.M743T) founder variant has been estimated to have arisen about 2500 years ago. ${ }^{23}$ This variant has been found in the homozygous state in Iranian Jewish ${ }^{7,22}$ and non-Jewish, ${ }^{22}$ Tunisian, ${ }^{32}$ Middle Eastern Muslim, ${ }^{38}$ Egyptian Muslim, ${ }^{39}$ and Japanese ${ }^{40,41}$ patients and in heterozygous state in a few patients from Italy, ${ }^{36}$ Tunisia, ${ }^{32}$ and Japan. ${ }^{42}$ The most prevalent variant in the Japanese ${ }^{41}$ and Korean ${ }^{33}$ patients is p.V603L, followed by p.D207V in Japanese and p.C44S in Koreans. Importantly, $13 \%{ }^{40}$ of all reported cases (and $50 \%$ of Japanese cases) ${ }^{41}$ with GNE myopathy carry at least one copy of p. V603L. Patients homozygous for this variant have a severe phenotype with earlier onset and faster progression of the disease. ${ }^{40}$

Most of the reported GNE myopathy variants in the GNE gene are missense. Only a few nonsense, frameshift, splice site variants, and indel variants ${ }^{31,40}$ have been described in GNE, of which none was found in the homozygous state, ${ }^{28}$ suggesting that this protein may have a critical role in embryonic development and viability, and that the total functional loss of GNE might be lethal in humans, as observed in mice. ${ }^{14}$

At amino acid position 277 of GNE, two other variants have been reported: p.R277W and p.R277Q. The p.R277W variant was identified in families of European ancestry, ${ }^{22,43,44}$ a family from China, ${ }^{43}$ a family of Italian descent, ${ }^{45}$ and a family from Japan). ${ }^{40}$ The c.830G $>$ A (p.R277Q) variant has been identified in the heterozygous state in patients from the Bahamas (with p.D256N), ${ }^{7}$ Taiwan (with p.I272S), ${ }^{46}$ Italy (with p.Q386_C388del/p.G352fs ${ }^{\star} 15$ ), ${ }^{47}$ and Japan (with p.D207V and p.V603L). ${ }^{40}$ This study is the first to report homozygous c.830G > A (p.R277Q) in GNE myopathy. Clinical variations were noted among our patients who were homozygous for the c.830G $>A$ (p.R277Q) variant and these previously reported cases. The average onset age of the patients in this study was $\sim 5$ years later than the 
previous cases. Although foot drop was the common initial presentation in all patients, three of our patients (with the c.830G $>$ A (p. $\mathrm{R} 277 \mathrm{Q}$ ) variant) exhibited hip-girdle weakness as well. The quadriceps muscles were involved in only one (25\%) of our patients (9a, Table 1) with this variant; however, still with less severity than other thigh and pelvic girdle muscles, whereas three of nine previously reported patients had affected quadriceps. One of the previous cases ${ }^{48}$ (Taiwanese-1, Table 1) had an incomplete right bundle branch block, but none of our or other previously reported patients had any cardiac abnormality. The involvement of upper limbs was more marked in the Taiwanese patients than that of the others. In our patients who were homozygous for the c.830G >A (p.R277Q) variant, mean CK values were lower than those in previously reported patients harboring the same variant, but in heterozygous state.

Currently, no clear genotype-phenotype correlations have been established for GNE myopathy. Varying clinical features in patients with different GNE variants suggest that different variants do not have equivalent functional impacts. Various clinical features associated with the same variant suggest the presence of other modifying gene variants, different genetic backgrounds, or epigenetic factors that might influence the clinical manifestations. In our analysis, we identified one novel variation that led to a synonymous codon change c.804G $>$ A $(p . L 268=)$ that potentially creates a novel splice site. The $\mathrm{G}>\mathrm{A}$ transition in the consensus splice site sequence (gene sequence) was predicted to alter splicing, by several splice site software programs thereby resulting in an altered enzyme structure and GNE myopathy.

Currently, there is no effective treatment for GNE myopathy; therefore the main focus of efforts is on prevention by prenatal carrier identification and genetic counseling for carrier couples. Recent preclinical studies with oral monosaccharides reversed the muscle hyposialylation in the GNE myopathy mouse model. ${ }^{49,50}$ A phase II clinical trial with oral sialic acid extended release tablets and two phase I trials with ManNAc are underway (www.clinicaltrials.gov).

In summary, clinical features of GNE myopathy in non-Jewish Persian patients from Iran were investigated and molecular analysis identified three disease-causing variants in the GNE: c.2228T >C (p.M743T), c.830G >A (p.R277Q), and c.804G >A (p.L268=). The latter is a novel variant that is predicted to cause abnormal splicing. The location of the most prevalent Middle Eastern variant, c.2228T >C (p.M743T), in the kinase domain and occurrence of the other two variants, c.804G $>$ A $($ p.L268 =) and c.830G $>$ A (p.R277Q), in the epimerase domain suggest that GNE myopathy families, even from the same ethnic group, can have different GNE variants. This is the first report of patients homozygous for c.830G $>$ A (p.R277Q) in the Persian population. Our findings, along with the other studies, emphasize the clinical heterogeneity of this disease. The results of this study expand the knowledge on the phenotype and molecular genetic heterogeneity of GNE myopathy, even in the non-Jewish Persian population.

\section{CONFLICT OF INTEREST}

The authors declare no conflict of interest.

\section{ACKNOWLEDGEMENTS}

We would like to thank Dr Hamidreza Haghighi for his useful comments. This study was undertaken as part of GENE-ME (a research initiative for investigation of inherited diseases from the Middle East).
1 Argov Z, Yarom R: "Rimmed vacuole myopathy" sparing the quadriceps. A unique disorder in Iranian Jews. J Neurol Sci 1984; 64: 33-43.

2 Sadeh M, Gadoth N, Hadar H, Ben-David E: Vacuolar myopathy sparing the quadriceps. Brain 1993; 116 (Pt 1): 217-232.

3 Nonaka I, Noguchi S, Nishino I: Distal myopathy with rimmed vacuoles and hereditary inclusion body myopathy. Curr Neurol Neurosci Rep 2005; 5: 61-65.

4 Krause S, Schlotter-Weigel B, Walter MC et al: A novel homozygous missense mutation in the GNE gene of a patient with quadriceps-sparing hereditary inclusion body myopathy associated with muscle inflammation. Neuromuscul Disord 2003; 13: 830-834.

5 Argov Z, Eisenberg I, Grabov-Nardini G et al: Hereditary inclusion body myopathy: the Middle Eastern genetic cluster. Neurology 2003; 60: 1519-1523.

$6 \mathrm{Lu}$ X, Pu C, Huang X, Liu J, Mao Y: Distal myopathy with rimmed vacuoles: clinical and muscle morphological characteristics and spectrum of GNE gene mutations in 53 Chinese patients. Neurol Res 2011; 33: 1025-1031.

7 Eisenberg I, Avidan N, Potikha T et al: The UDP-N-acetylglucosamine 2-epimerase/Nacetylmannosamine kinase gene is mutated in recessive hereditary inclusion body myopathy. Nat Genet 2001; 29: 83-87.

8 Kayashima T, Matsuo H, Satoh A et al: Nonaka myopathy is caused by mutations in the UDP-N-acetylglucosamine-2-epimerase/ $\mathrm{N}$-acetylmannosamine kinase gene (GNE). J Hum Genet 2002; 47: 77-79.

9 Hinderlich S, Stasche R, Zeitler R, Reutter W: A bifunctional enzyme catalyzes the first two steps in $\mathrm{N}$-acetylneuraminic acid biosynthesis of rat liver. Purification and characterization of UDP-N-acetylglucosamine 2-epimerase/Nacetylmannosamine kinase. J Biol Chem 1997; 272: 24313-24318.

10 Stasche R, Hinderlich S, Weise $\mathrm{C}$ et al: A bifunctional enzyme catalyzes the first two steps in $\mathrm{N}$-acetylneuraminic acid biosynthesis of rat liver. Molecular cloning and functional expression of UDP-N-acetyl-glucosamine 2-epimerase/Nacetylmannosamine kinase. J Biol Chem 1997; 272: 24319-24324.

11 Kelm S, Schauer R: Sialic acids in molecular and cellular interactions. Int Rev Cytol 1997; 175: 137-240.

12 Ulloa-Aguirre A, Timossi C, Damian-Matsumura P, Dias JA: Role of glycosylation in function of follicle-stimulating hormone. Endocrine 1999; 11: 205-215.

13 Horstkorte R, Nohring S, Wiechens $\mathrm{N}$ et al: Tissue expression and amino acid sequence of murine UDP-N-acetylglucosamine-2-epimerase/ $\mathrm{N}$-acetylmannosamine kinase. Eur J Biochem 1999; 260: 923-927.

14 Schwarzkopf M, Knobeloch KP, Rohde E et al: Sialylation is essential for early development in mice. Proc Natl Acad Sci USA 2002; 99: 5267-5270.

15 Weidemann W, Stelzl U, Lisewski $U$ et al: The collapsin response mediator protein 1 (CRMP-1) and the promyelocytic leukemia zinc finger protein (PLZF) bind to UDP-Nacetylglucosamine 2-epimerase/N-acetylmannosamine kinase (GNE), the key enzyme of sialic acid biosynthesis. FEBS Lett 2006; 580: 6649-6654.

16 Huizing M, Rakocevic G, Sparks SE et al: Hypoglycosylation of alpha-dystroglycan in patients with hereditary IBM due to GNE mutations. Mol Genet Metab 2004; 81: 196-202.

17 Ricci E, Broccolini A, Gidaro T et al: NCAM is hyposialylated in hereditary inclusion body myopathy due to GNE mutations. Neurology 2006; 66: 755-758.

18 Hinderlich S, Salama I, Eisenberg I et al: The homozygous M712T mutation of UDP-Nacetylglucosamine 2-epimerase/ $\mathrm{N}$-acetylmannosamine kinase results in reduced enzyme activities but not in altered overall cellular sialylation in hereditary inclusion body myopathy. FEBS Lett 2004; 566: 105-109.

19 Broccolini A, Gidaro T, De Cristofaro R et al: Hyposialylation of neprilysin possibly affects its expression and enzymatic activity in hereditary inclusion-body myopathy muscle. J Neurochem 2008; 105: 971-981.

20 Tajima Y, Uyama E, Go S et al: Distal myopathy with rimmed vacuoles: impaired O-glycan formation in muscular glycoproteins. Am J Pathol 2005; 166: 1121-1130.

21 Desmet FO, Hamroun D, Lalande M, Collod-Beroud G, Claustres M, Beroud C: Human Splicing Finder: an online bioinformatics tool to predict splicing signals. Nucleic Acids Res 2009; 37: e67.

22 Darvish D, Vahedifar P, Huo Y: Four novel mutations associated with autosomal recessive inclusion body myopathy (MIM: 600737). Mol Genet Metab 2002; 77: 252-256.

23 Askanas V, Engel WK: Muscle Aging, Inclusion-Body Myositis and Myopathies. Oxford, UK: Wiley-Blackwell. 2012.

24 Nishino I, Noguchi S, Murayama $\mathrm{K}$ et al: Distal myopathy with rimmed vacuoles is allelic to hereditary inclusion body myopathy. Neurology 2002; 59: 1689-1693.

25 Nalini A, Gayathri N, Dawn R: Distal myopathy with rimmed vacuoles: report on clinical characteristics in 23 cases. Neurol India 2010; 58: 235-241.

26 Khademian H, Mehravar E, Urtizberea J et al: Prevalence of GNE p.M712T and hereditary inclusion body myopathy (HIBM) in Sangesar population of Northern Iran. Clin Genet 2013; 84: 589-592.

27 Mitrani-Rosenbaum S, Argov Z, Blumenfeld A, Seidman CE, Seidman JG: Hereditary inclusion body myopathy maps to chromosome 9p1-q1. Hum Mol Genet 1996; 5: 159-163.

28 Malicdan MC, Noguchi S, Nishino I: Perspectives on distal myopathy with rimmed vacuoles or hereditary inclusion body myopathy: contributions from an animal model. Lack of sialic acid, a central determinant in sugar chains, causes myopathy? Acta Myol 2007; 26: 171-175.

29 Park YE, Kim HS, Choi ES et al: Limb-girdle phenotype is frequent in patients with myopathy associated with GNE mutations. J Neurol Sci 2012; 321: 77-81. 
30 Huizing M, Krasnewich DM: Hereditary inclusion body myopathy: a decade of progress. Biochim Biophys Acta 2009; 1792: 881-887.

31 Hinderlich S, Weidemann W, Yardeni T, Horstkorte R, Huizing M: UDP-GIcNAc 2 epimerase/ManNAc kinase (GNE): a master regulator of sialic acid synthesis. Top Curr Chem 2013; e-pub ahead of print 11 July 2013.

32 Amouri R, Driss A, Murayama K, Kefi M, Nishino I, Hentati F: Allelic heterogeneity of GNE gene mutation in two Tunisian families with autosomal recessive inclusion body myopathy. Neuromuscul Disord 2005; 15: 361-363.

33 Sim JE, Park HJ, Shin HY, Nam TS, Kim SM, Choi YC: Clinical characteristics and molecular genetic analysis of Korean patients with GNE myopathy. Yonsei Med J 2013, 54: $578-582$

34 Milman Krentsis I, Sela I, Eiges R et al: GNE is involved in the early development of skeletal and cardiac muscle. PLoS One 2011; 6: e21389.

35 Sivakumar K, Dalakas MC: The spectrum of familial inclusion body myopathies in 13 families and a description of a quadriceps-sparing phenotype in non-Iranian Jews. Neurology 1996; 47: 977-984.

36 Broccolini A, Pescatori M, D'Amico A et al: An Italian family with autosomal recessive inclusion-body myopathy and mutations in the GNE gene. Neurology 2002; 59: 1808-1809.

37 Diaz A, Montfort M, Cormand B et al: Gaucher disease: the N370S mutation in Ashkenazi Jewish and Spanish patients has a common origin and arose several thousand years ago. Am J Hum Genet 1999; 64: 1233-1238.

38 Eisenberg I, Grabov-Nardini G, Hochner $\mathrm{H}$ et al: Mutations spectrum of GNE in hereditary inclusion body myopathy sparing the quadriceps. Hum Mutat 2003; $21: 99$

39 Grandis M, Gulli R, Cassandrini D et al: The spectrum of GNE mutations: allelic heterogeneity for a common phenotype. Neurol Sci 2010; 31: 377-380.

40 Cho A, Hayashi YK, Monma K et al: Mutation profile of the GNE gene in Japanese patients with distal myopathy with rimmed vacuoles (GNE myopathy). J Neurol Neurosurg Psychiatry 2013; 85: 914-917.
41 Mori-Yoshimura M, Monma K, Suzuki N et al: Heterozygous UDP-GIcNAc 2-epimerase and $\mathrm{N}$-acetylmannosamine kinase domain mutations in the GNE gene result in a less severe GNE myopathy phenotype compared to homozygous $\mathrm{N}$-acetylmannosamine kinase domain mutations. J Neurol Sci 2012; 318: 100-105.

42 Tomimitsu H, Shimizu J, Ishikawa K, Ohkoshi N, Kanazawa I, Mizusawa H: Distal myopathy with rimmed vacuoles (DMRV): new GNE mutations and splice variant. Neurology 2004; 62: 1607-1610.

43 Sparks SE, Ciccone C, Lalor M et al: Use of a cell-free system to determine UDP-N-acetylglucosamine 2-epimerase and $\mathrm{N}$-acetylmannosamine kinase activities in human hereditary inclusion body myopathy. Glycobiology 2005; 15: 1102-1110.

44 Saechao C, Valles-Ayoub Y, Esfandiarifard S et al: Novel GNE mutations in hereditary inclusion body myopathy patients of non-Middle Eastern descent. Genet Test Mol Biomarkers 2010; 14: 157-162.

45 Stober A, Aleo A, Kuhl V et al: Novel missense mutation p.A310P in the GNE gene in autosomal-recessive hereditary inclusion-body myopathy/distal myopathy with rimmed vacuoles in an Italian family. Neuromuscul Disord 2010; 20: 335-336.

46 Chu CC, Kuo HC, Yeh TH, Ro LS, Chen SR, Huang CC: Heterozygous mutations affecting the epimerase domain of the GNE gene causing distal myopathy with rimmed vacuoles in a Taiwanese family. Clin Neurol Neurosurg 2007; 109: 250-256.

47 Broccolini A, Ricci E, Cassandrini D et al: Novel GNE mutations in Italian families with autosomal recessive hereditary inclusion-body myopathy. Hum Mutat 2004; 23: 632.

$48 \mathrm{Li} \mathrm{H}$, Chen $\mathrm{Q}$, Liu $\mathrm{F}$ et al: Clinical and molecular genetic analysis in Chinese patients with distal myopathy with rimmed vacuoles. J Hum Genet 2011; 56: 335-338.

49 Niethamer TK, Yardeni T, Leoyklang P et al: Oral monosaccharide therapies to reverse renal and muscle hyposialylation in a mouse model of GNE myopathy. Mol Genet Metab 2012; 107: 748-755.

50 Malicdan MC, Noguchi S, Hayashi YK, Nonaka I, Nishino I: Prophylactic treatment with sialic acid metabolites precludes the development of the myopathic phenotype in the DMRV-hIBM mouse model. Nat Med 2009; 15: 690-695.

Supplementary Information accompanies this paper on European Journal of Human Genetics website (http://www.nature.com/ejhg) 